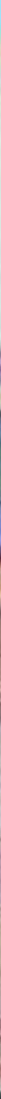




\section{La pintura indianista. José Sabogal en la Argentina}

Roberto Anigo

Lhiversidad de Buenos Ares de Argentina

La etapa argentina de Jose 5abogal (Cajabamba, $18 B 8$ - Lima, 1956) es un momento poco estudiado de su trayectoria; sin embargo es clave para comprender la génesis del indigenismo. Dos momentos son relevantes: la etapa de formación en Buenos Aires y Jujuy. con la elección de un lenguaje artístico, y la exposición celebratoria de 1928. En la historia del arte argentino su presencia ha pasado casi inadvertida, sin embargo la recepción de su obra, las redes de artistas que integro, permite pensar desde otro ángulo la habitual polaridad entre la tradición y la nuevo, además de explorar las redes regionales más que las experiencias con la modernidad europea.

\section{José Sabogal / arte peruano / arté argentíno / indigenismo / nacionalismo}

The argentinean stage of José Sabcgal (Cajabamba, 1888 - Lima, 1956) is an understudied moment of his career, however, is key to understanding the genesis of Indianism. There are two relevant moments: the formation stage in Buenos Aires and Jujuy, with the choice of an artistic language; and the exhibition of 1928, In the Argentinean art history his presence has been almost unnoticed, however the reception of his work, the artists networks he integrated, alows the thought of another angie to the usual polarity between tradition and the new, moreover to explore the regional networks over the experiences with European modernity.

José Sabogal / Peruvian art / Argentinean art/indigenism / nationalism

\section{Primerit parte: la formación}

José Sirbogal (Cajabamba, 1888 - Lima, 1956) llegó a Bvenos Aires en fecha dificil de precisar con exactitud', Las referencias secundarias difieren al respecro, auncue probablemente haya sido a fines de 1911 o comienzos del ano siguiente? . En su estadia europea habia frecuentado, entre 1909 y 1911, la Escuela Libre de Desnudo y la Academia de España en Roma, donde debe haber adquirido las primeras herramientas para la composición de

Agradecoa las comentarios y la documentacióe de anchivo facilirada por Natalia Majlaf.

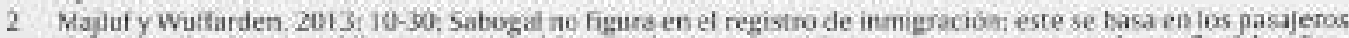
6o tarces de utramas, al llegar ee velero hizo oscala en Mónteviceo y no se conservan registros fluviales 9ara

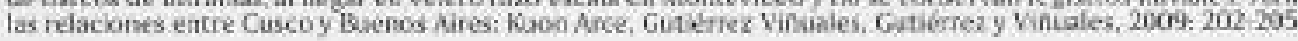




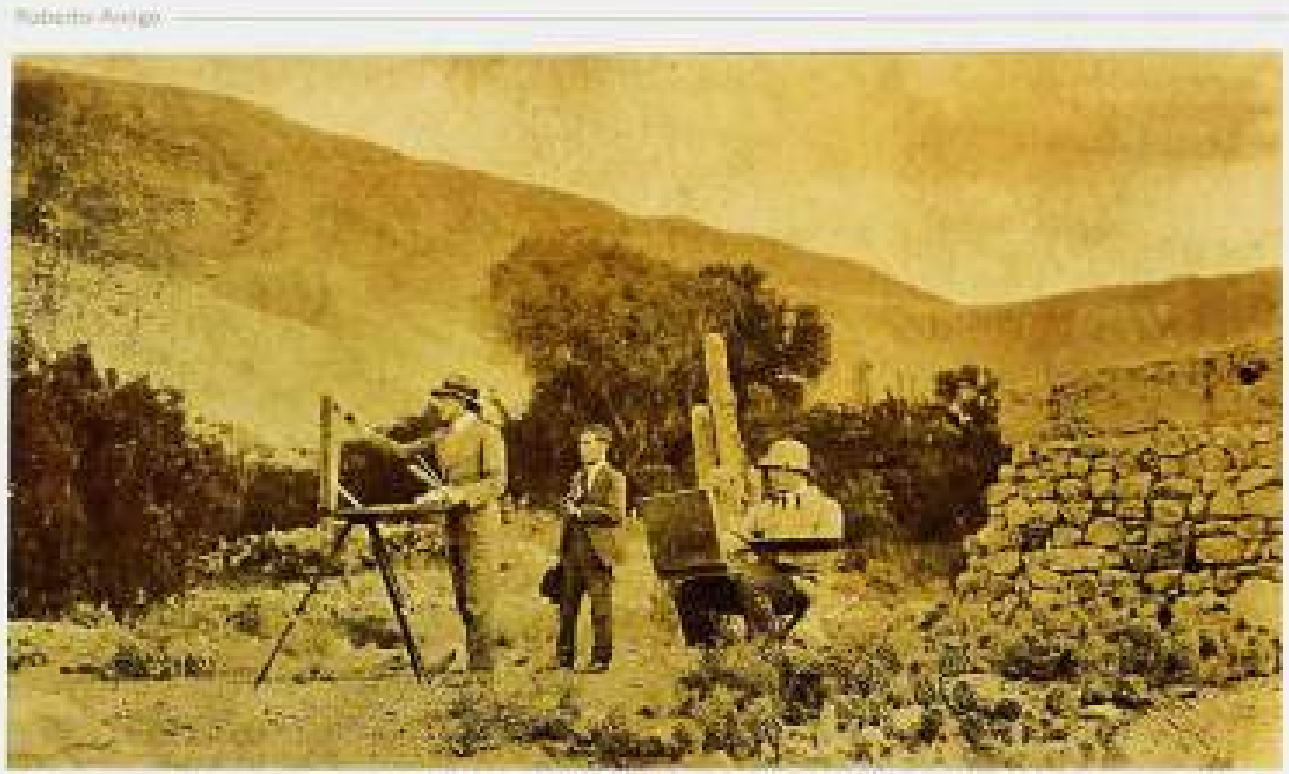

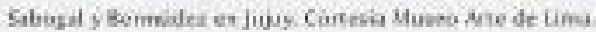

las figuras, segün las formulas del naruralismo finisecular. Luego de realizar el habitual grand-tour por el norte de Áfrici y un tiempo breve en Espana se embarcó como grumete en un velero hacia Buenos Aires. Es posible que haya praccicado la representación de tipos exóticos desde el arientalismo tardio, que el viaje por el Mediterràneo presupone para todo artista que io emprende. A este escaso bagaje se suma la observacion de los costumbrismos regionalistas, cuyo exito notable de menado cosnprobó en Buenos Aires, principat] boca de venta en el extranjero de los talleres espanoles.

La celebrada Exposicion internacional de Arte de 1910 no solo habia agudizado fa rencion no resueta entre lo nacional y lo internacional, sino que también hizo notable la fractura entre los at tistas de le generación decimonónica y de la camada del nuevo siglo que comenzaba a dominar los aparatos institucionales. Asf, los artistas descendientes del viejo patriciado y del comercio con ultramar se llamaban a tetiro, aceptando destimos diploma. ticos o la callada convivencia con los artistas hijos de la inmigración. Sabogal, entonces. Se inserta como estudiante, en un momento dawe: fue testigo del desplazamiento social y generacional dentro del arte argentino. Estos desplazamientos no ocurrieron sin disputa. se expresaron con virulencia sarante la reforma de la educación plastica propuestit por Pio Collivadino en 1908. El nuevo director de la Academia Nacional de Bellas Artes estaba dispuesto a conducifla con mano de hieso es cuanto a horarios, asistencia y durzza en los examenest. Emesto de la Carcova y Fduardo Sfyori, los amables maestros decimononicos renunciaron: el arte como pasatiempo había terminadis. La enschanza se dirigio a forma profesionales no solo en pintura y escultura, sino rambién en las artes decofativas e industriales. 11 dibujo era la herramienti fundamental, con las obligadas copias de sesos, ejercicios de sombreado y de perspectiva, para finalizar con la aplicación de color, modelo svo

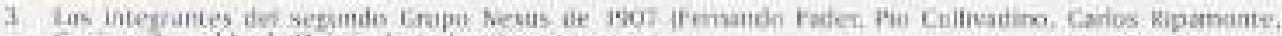

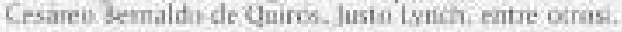

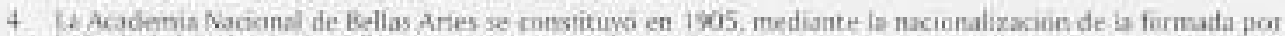

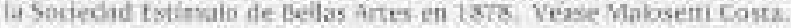


y paisaje al aire libre, 5abogal se incarporó probableatente en los affos superiores taícomo permitia el nuevo reglamento; seguramente Colivadino avaló el tiempo de estudios en Roma, ya que el mismo se hahia fornado en Inalia entre 1890 y 1906 . L a mayor amistad on la Academai la estabiccio con el unguayo Modesto Luccioni, artista de escasa relevancia que apenas fogeró que se aceptara ałguna obra suya en el salón.

Sabogal caindo obrivo el título de prnfesor de dibajo aceptó un cargo docente en una escuela de la provincia norteña de Jujuy que ocupó entre 1913 y 1917 . Era un momento clave para ser docente de dibujo, ya que habian impactado los debates renowadores en su enseranza impalsados por la prédica de Marrín Malharro: la obligatoriedad de la ensefiarza del dibujo, tanto mediante leccioaes pautadas como cort el dibujo libre frente a la naturaleza, desarrollaba la petcepción y cjercitaba la imaxinacón del nino, respetando las individualidades, $A$ su muerte en 1911. lo sucediö su discipulo Walter de Navazio. can quién Sabogal ha tenida seguro contacto: lo retrato al lapiz con su aire melanecilica y aristocrático, cuando obtuvo uno de los premios de pincura en el Salon Nacional de 1913.

Sabogal, ademis del dibujo, necesariamente tawo que estar al tanto de Jos desafios planteados a la educación: debía ser el instrumento para armonizar los elementos cosmopolitas de la inmigración en una nación homogenea. Para ello. Ricardo Rojas propuso que desde ef sentido histúrico se promoviera "un esfuerzo generoso y conscientemente realizado en frovor del territorio, del idioma, de la tradición o de la hegensoaia futura del pais", para ello había que regenera el "patriotismo instintivo mediante "la restauración del espintu indagena que la civilizeciuin clebe sahvar en todas partes por razones estéticas y religjosas. "En Blasoin de plote. 1912, profundiza la idea del indianismo: instinto colectivo que proviene de la naturaleza, categoria que reemplaza la dicotomia covilización y barbarie. Otro aspecto de estas ideas circulantes era la oposición del cosanopolitismo de la gran urbe frente a la vida en las provincias del interior, que eran el reservorio de la tradición, del alma de la tierra argentina en doade perduraba la raíz hispana, cuestión expresada es las novelas y ensayos de Manuel Gálvez, tanto en El diorio de Gobrid Quimea, 1910, como en El solar dé lo razo, 1913". Este programa de nacionalismo modemo se levaba a cabo ent el arte medianse- la representa. ción de los paisajes y tipos, tal como aconsejó al pintor José A. Merediz;

"É debe ahora realizar en nuestros viejos pueblos en jujuy, en Salta, en Catamalca lo que ha hecho en Segovia y Toledo. Sera asi el revelackor de. nuestro paisaje de provincia. En aquellas ciudades pobres, humildes. silenciosas, trodavia coloniales, vive of alma de nuestra raza",

Asi. Sibogal se forno bajo el impacto de las ideas nacionalistas que determínaban cuál debia ser el arte argentino. Recibió, sin dads, la confirmación de tal derrotero por los

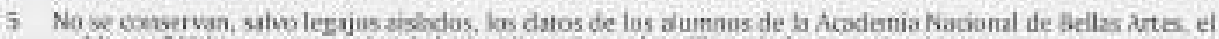

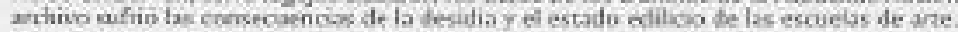

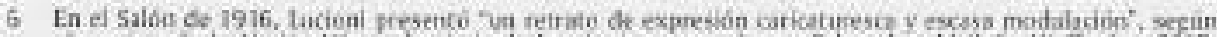

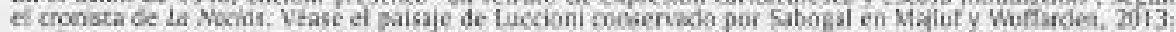

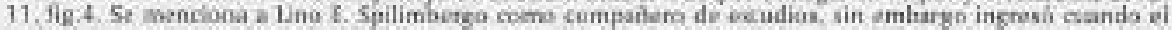

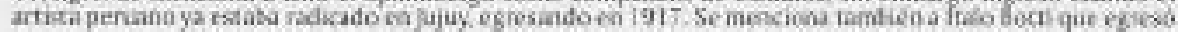

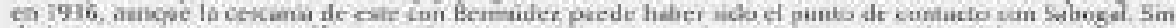

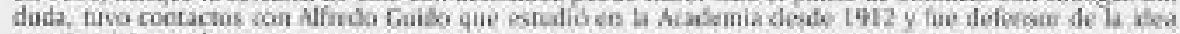
moncunista en el arte.

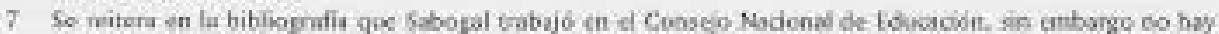

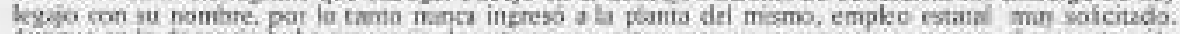

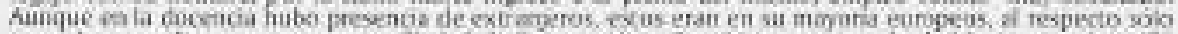

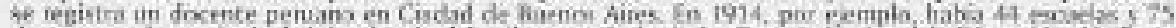

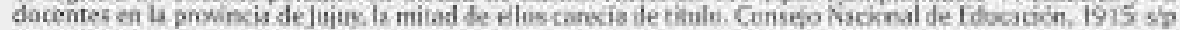

( ) Kojas. 1909 360.

9 Mhas -2012.

10 Midon. 
logros que obtenian los defensores de esa estética en el Sabōn Nacional, como el italo. argentino Pompeso Boggio que obtuwo el primer premio por Fipos quichuas de la Qunbrado de Humahunca en 1912. probablemente el primer salón oficial que observo el pintor peruano en Buenos Aires. Estos salones amuales iniciados en 1911. fueron el espacio de la legitimacion del arte nacional, que era comprendido como la representación de los tipos. las coscumbues y, principalmente, los paisajes stranos o nurfentos que desplazaron la represeratación de la pompe, hezemònica en el sigglo anterior. Formalmente la modernidad se adjudicaba a las recetas postimpresionistas, con paletas luminosas y yuxtaposicion de pinceladas; a la renovación cromatica del naturalismo, y a la disolacion del yo en ha naturalexa cuyo resultado fue una pintura melancolica. de tintes grises y violaceos. Tendencias que compartian la práctica del plein ari, cue además de condición creativa era la expresión de la conciencia de la naturaleza, la verdad del territorio de la nacion.

En Jujuy, el destino docente de Sabogal, se encontraba radicado José Antonio Terry. En 1910 emiaba a los saloxes pintura de ripos regionales como Tipe solamunonetio y Tipo napolitano realizados er su estadia europez, Por este dominio del género fue convocado. junto al mencionado Pompeyo Boggio, al vaje arqueológico del Museo Etnográfico de la Universidad de Buenos Aires por el norceste argentino. Terry, finalinente, se estableció en Tuleara, donde se dedicó a la representación de los tipos lugarenos. Además de los con: tactos con Terry, el jorien Sabogal redbió la influencia mayor de jorge Bermúdez, artista ya consagrado Aruncino nojo había obtenido el premio en el Salón Nacional de 19131 formado con el célebre español Ignacio Zulosga. A los pascos conjuntos se sumaba otro artista aficionado Emilio Kunz". La paleta de Bermodez se hizo más luminosa cuando recorrió las provincias de Catamarca y Jujuy eatre 1914 y 1915, marcada por el sol del norte. Antes de partir de la Argentina, Sabogal pudo presencia el nuevo éxito de Bermúdez: en 1917 obtuvo el Premio hdquisición cn el Salón Nacional por Rlăa de gulias, sa obta más compleja por el movimiento de les figuras, alejúndose de la sensación de estatismo de su pintura precedente" La consolidarión definitiva de Rermúdez ocurrio con la exposición de 1923 en la prestigiosa galeria Witcomb: Ricardo Rojas lo definió como "el pintor de la raza". Un proceso similar ocurria en Lima con Sabogal. Sin embargo, el provecto de escuela nacional que marcaba la obra de Bermudez quedó trunco con su regreso a Granada y su temprana muerte's ef lugar vacante lo pcupo, en 1928, Cesáreo Bernaldo de Quirós con los gauclsos federales del litoral. Quirós, al igual que Terry y Bermúdez, se liabia formado con la pintura de tipos regionales europeos, las huellas del regionalismo espentol en la pintura de Sabogal tienen sti origer en estas mediaciones del arte argentino.

La consagración de un artista dependia de la suerte corrida en el salón anual, que garantizaba la inserción al circuito comercial de galerias. Ser aceptrido era el primer éxito. porque la admision dependia del jurado, siempre atenido a un estricto reglamento que controlaba la Comisión Nacional de Bellas Artes ${ }^{14}$. Sabogal realizó el recorrido habitual de la Academia al Salón: su primer ingreso lo loggó en el IV Salón Nacional de Bellas Artes, de 1914, con dos oleos de paiszje del noroeste argentino. La critica en el diario Lo Nacín fue tan breve como lapidaria: "Inucilizada tela Lha calle de fajuy de José Sabogal." Este Salón adquirió relevanda por la negativa de Fernando Fader a aceprar el premio de tres mil pesos otorgado

It Mailufy Wuffarden, 20t3: 15

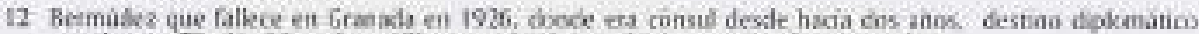

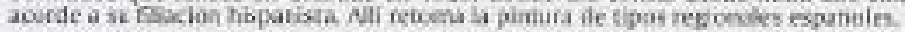

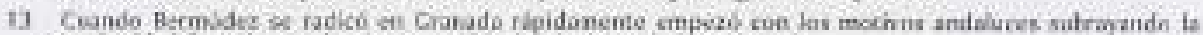

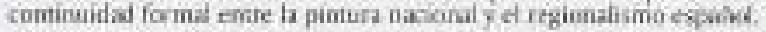

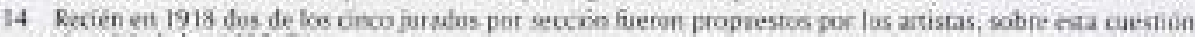
nuse Wocleter 1909 isa. 


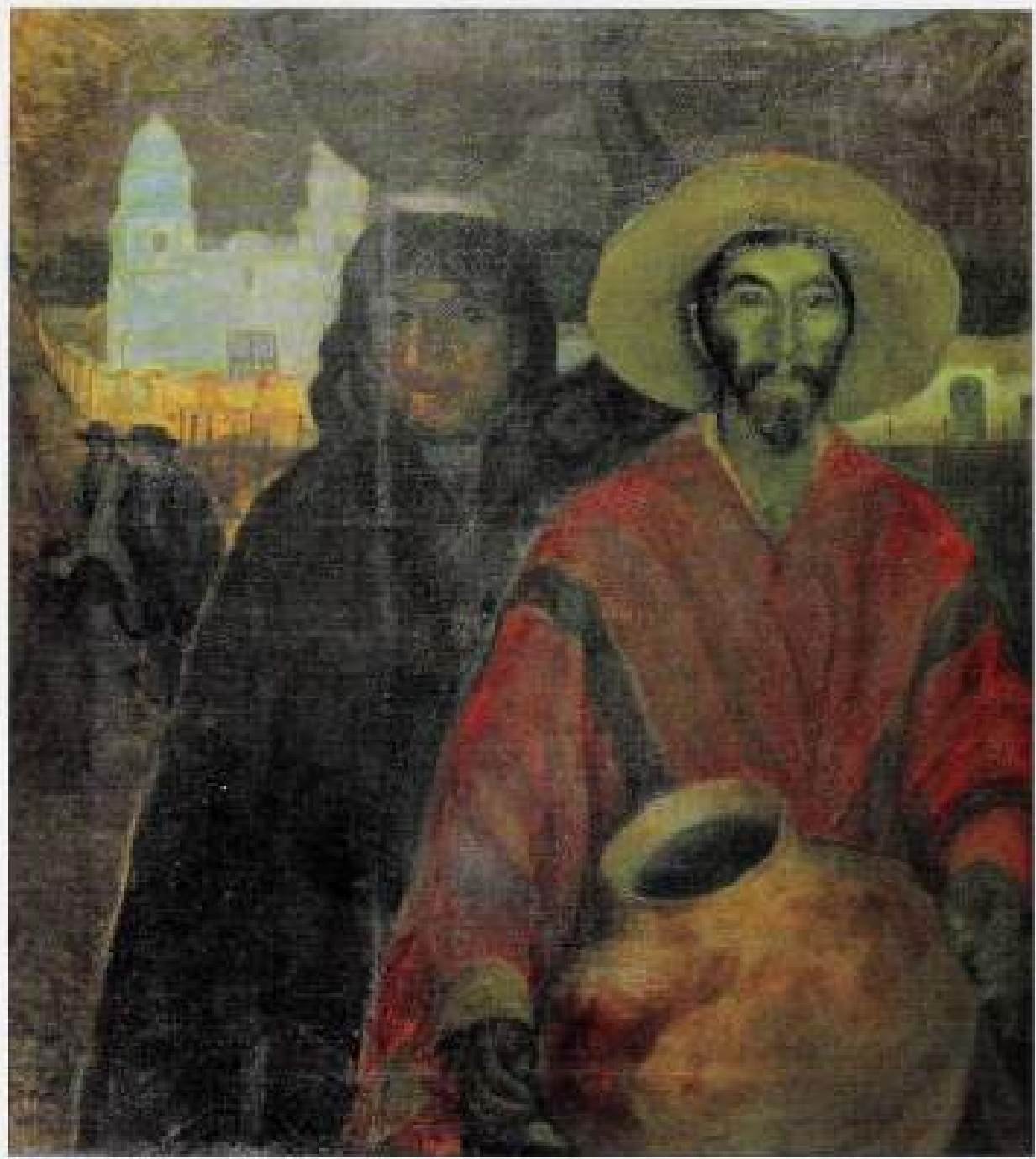

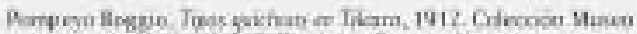
vacbone de Betin Artes tuecos aire

por el jurado a Mantontes de diraha, ya que habia tosado su obrd al doble de valor ${ }^{15}$. Este precio superlativo no puede ser usado como parámetro para la obrz de sabogal que tasa sus abras entre los precios más bajes: Uno calle de lyjur a doscientos pesos y Berrancas del Kín Gindé doscientos cilicueata pesas, Por otra parte, el articuls 24 del reglamento dejaba fuera de concurso a Sabogat, ya que estipulaba que la recompensa se otorgaha únicamente a arsistas argentinos y de "preferencia a las que tengan caracter nacional".

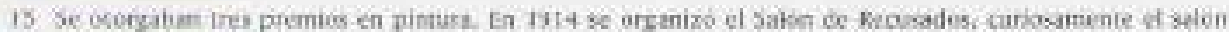
docial fue el inas mimeroso de la décacta con 440 obras 


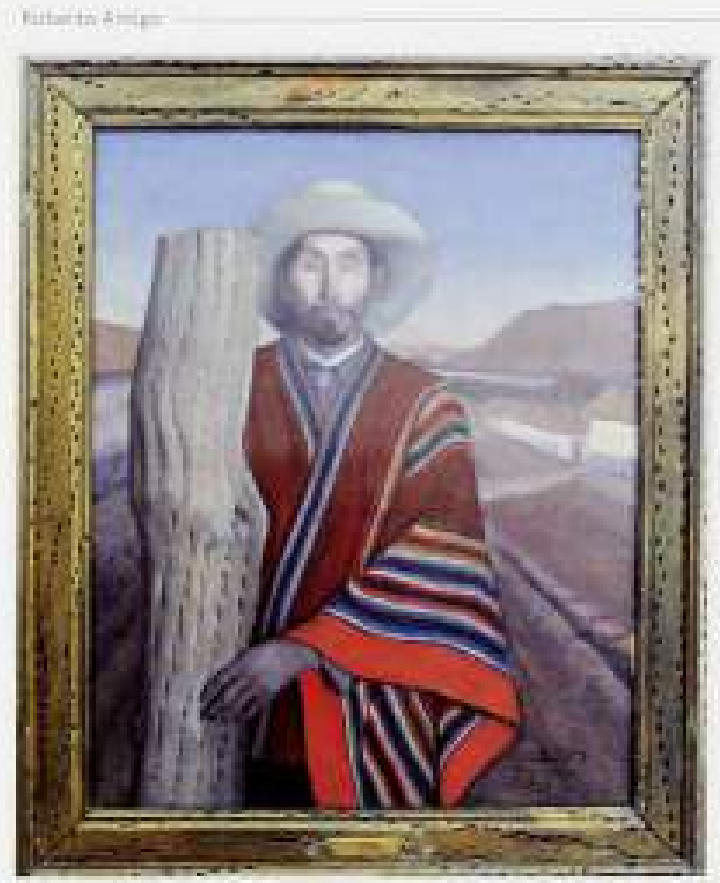

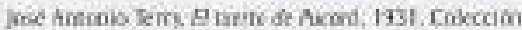

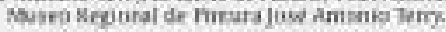

La noticia de la actwidad de sabogal llege a Lima por usa nota firnada por Teofilo Custillo en 1916, cuan do aquel expuso por primera ver de manerz individual en la Biblioteca Popular sle Sin Saluadar de Jujuxy" In rotal presentó cuarenta telas, entre ellas Gucho jujetio ise mezciona como envio al Satón Nacictal del ano anteriot), sin embargo no figura en el catalogo, a no ser que refiera a Sosingo, motivo de Tilcara cuyer tirulo peede referir a la calma norteña o a un lipo popular ${ }^{2}$, Las obras de esta exposición fueron er si maryoria paisajes que permiten seconstruir el mapa de la zona: ef arrista fecorno la Quebrada de Humahuaca, Río Grande. Tileara, Purnaraarca y La Tabloda. Este panorama del territorio era acompañado por las vistics de edificous colooiales, también de la ciudad de Jujuy in septiembre de 1917 montó un nueva exposición màs reducida con "retratos, una serie

de caticaruras $y$ un grupo de paisajes y escenas de costumbres, entre los que resalta una tela titulada Girayritos. ${ }^{-i k}$

La obra de Sabogal en la Argentina se encuentra dispersa, pero las conocidas denotan la influenca de Bermudez en los tipos, pero una mayor independencia en la construccion de algunos passajes sin figuras, como cantain solitario, que se asocia con la tendencia haxia tua mayor sintesis y variación tonal. Otro paisaje Jocalizado presenta un gran sauce, con caserio y montanas de fonde, en este caso pudierz ser atribuido a un intists argentino del periodo sin inconvenientes. Sabogal transita el camino del naturalismo sât sutilezas me. lancolicas; acepta la ptemisa de la verdad de la naturaleza; aquello que tiene la obligación de captar el artista: por eso opta por la representación objetiva, que pernits al espectador aproximarse a ese ideal espiritual que la tierra encierra, más que a la intelectual combinación de visión y sentimiento. tan en boga en los artistas de su generación. Asi. La pintural transmite el inensaje del territorio predestinado, donde a diferencia de la gran arbe predominaba incormpio lo autoctono. La diferencial radical es la centralidad en el ferú de lit śascusion por la tierra que anula la posibilidad del paisaje, aun más coma emıfüfítiong. La elecciba de la pertenencia a la corriente de Bermúdez y Terry permitió a Sabogal su trans. posiciox, ya que la objetividad naturalista facilitaha la cleriva hacia la monumentalidad, ef realismo o la sintesis figurativa.

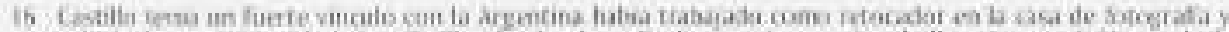

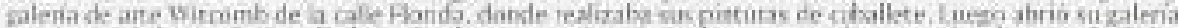

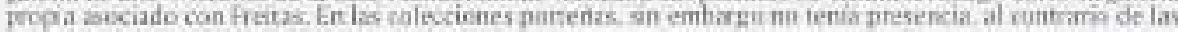

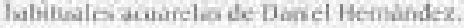

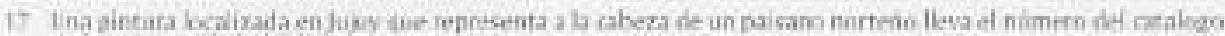
Le la muestia de la bihisoteca.

is Mailut y Wuttanden. $2012-15$. 
La pricridad de la pintura de "carâcter nacional' para los premios del salón fue tin es. timulo para el desarrollo de lo que se denomina "nativismo". Marta Penhus ha defisido esta cacegoria como referencia "a un conjunto de valores vinculados a la tierra y a la tradicion, identificaban contenidos e intenciones artisticas y, asimismo, legitimaban el presente al incluirlo en una genealogia históricat" El nativismo en las artes plásticas es la representación de los hombres en la tiesra, que es naturaleza y cultura, ya que aquellos portan la fuerza de lo telúrico desde el mestizaje, resultado de la bistoria. La utilidad del termino radica en que permite tomar distancia del indigenismo, cuyas bases ideologicas están mareadas por la plástica mexicana, y cuyo impacto regional es postirior. Es interesante apuntar que Jose Maria Ramos Mejia, a cargo del Consejo Nacional de Educación, sostenia que hacian falta dos generaciones de mestizaje para regenerat la raza que babía sufrida la influencia migratoria². Desde ya, al ingresar en los añog veínte el nativismo toma un carácter màs tradicional y visualmente conservador frente a la llegada de la modernización vanguardista. Tal vez sea necesario una mayor distinción dentro del nativismo fo abandonar este término cargado de connotaciones xenófobas que piseden llevar a confusión, ya que st tso local es asimilacionista, vinculado a li idea de mestizaje más que de esenciz de la raza? ), La pintura de tipos y costumbres norterios pueden nominarse como "indaanismo", segun el término cultural usado por Kicardo kojas en Blason de plate y profundizado en Eurindie, más atin cuando indianismo es a la yez tradición y destion. Asi, bo es sóle una rememoracion conservadora sion un programa para constituir la identidad, un destino espiritual que debe lograr capturar a las masas inmigratoria5. Superar la raza como factor material de la civilización implica en Rojas, como ha señalado Graciela Ferrás.

... compremder en ef 'indio' raices peovenientes de las grandes civiliza: ciones del mundo. que confluyen en la elaboración de un tipo autóctone americano que encama una dimensión unwersal y que, a su vez, constitiye la base de ntestra nacionalidad. Al mismo tiempo que podria pensarse que la nacionalidad tenia un sustrato étnico en el indio, la idea de crisol de razas disolvia este sustrato en una dialéctica abierta de las razas 'pon venir' que encontraba su sentido mis intimo en su facricidad 'todo' es argentino en esta tierra, todo elemenzo extrinseco pasa a ser "otra cosa. al pisar este suclo: pasa a ser 'argentino' ?

E indianisno es también una manera de definir el sentimiento criollo por la emoción del ternitorio, por eflo la preocupación constante de Rojas por las manifestaciones estéticas. Es la cuestion inmigratoria la que separa radicalmente las kccuros de representaciones similares en la Argentina y en ef Pertis asi Sabogal necesariamente debe abandonar la definición estilistica de nativismo o indianismo, para capturar la de indigenisano que le permite rearmarse luego de sa viaje a México. Sabogal es entendido como "pintor d́e indios", pero su mirada $\rightarrow$ a diferencia del muralismo mexicano no es historicista, aunque deterido en el tiempo y por lo tanto, en su casácter esencial, invariable y resistente a la modemidad ${ }^{2}$. La experiencia mexicana, por otra parte, fue un estimulo a su interés por las creaciones de la cultura popula, que era menor entre los mativistas argentinos. Es la staperposición de]

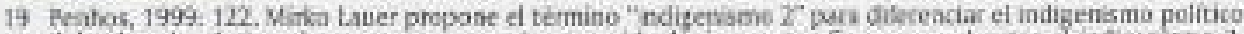

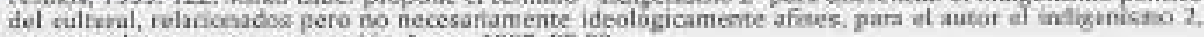
conceptoslitience. es una revesion. Easer. 1997:25.12

20) Cr. Nuiferde 2003.58 .

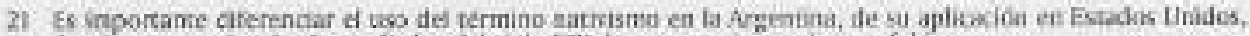

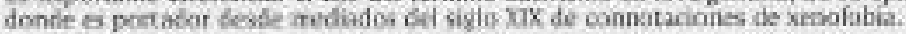

22 ferds, 200 a 13.

2) Majlaf y Wiffarden, 2012:14 
discurso mexicano sobre el aprendizaje argentino, lo que permite a Sabogal diahogar con la posición de intelectuales y literatos que generaron el marco de discusión sobre que es la nación en el Peri.

Sabogal expuso nuevamente en el Salón Nacional en 1918, ya de fegreso en Buenos Aires: como si preparase el regreso el envío consta de dos obras Tarde jujelia y Limela. Esta ultima es reproducida en el catálogo: la figura ceatral de una tapada mirando sedectoramente al espectador, con el fondo de arquitectura limena y sus caracteristicos baicones. Recuerdo a la distancia que sefiala el fin de los motivos regionales argenfinos yanuncio de sus motivos criollos.

Ademis del ervio al Val Salòn también expuso con la Sociedad Nacional de Artistas Pintores y Escultores (el núcleo de esta asociación lo formaban los Artistas del Pueblo) que convocb al Primer Salón "sin jurados y sin premios" "24, En esta ocasión, Sabogal presentó una tela con mayor presencia de ripos poptulares: Camavol de Tilcara. Es, sin duda. la mejor obra conocida de su etapa argentina, tanto ea la soltura compositiva como en el manejo cromático. En este contra-salón confluyeron artistas de distintas tendencias estéricas reproduciendo la misma visibilidad del salón, sin embargo el sentido ya no era la competencia y la legitimación sino ia convicdón del arte como comunidad de trabajo. De este modo, Sabogal se relacionó con los artistas anarquistas que proponian el modelo de artista-proletario, la sindicalizacions gremial y la difision popular del grabado. Sin embargo, la experiencia de Sabogal en el norte argentino, Salta y Jujuy, lo relacionaba con orra realidad social, distante de la cultura radical de los inmignantes de los barrios porfeños

La figura de Sabogal permite pensar los grises entre el nacioaalismo artistico, por un lado. y el nucleo duro de los artistas de izquierdas. Esto obliga a pensar la temprana relación de la representación erudita de la cultura popular de base campesina -cuyo mejor exponente fue Alfredo Gramajo Gutiérrez. en el salon oficial del año 1918-con los programas estéticos de las izquierdas. Sintomáticamente Sabogal presenta una escena de carnaval popular y campesino, ies otra de las puertas que abre antes de su definitiva partida? Desde las ideas de Proudhon ya lo habia argumentado Martín Malharro-era posible defender el arte nacional como participe de las emociones del pueblo, y desde alli validar la representación de la geografia en que se fusionaban hombres, costumbres y naturalezas.

Otro aspecto interesante es la revaloración fuerte del grabado en la segunda mitad de la década de 1910, tanto por los mencionados Artistos dei Puebla -que utilizaban preferentemente el aguafuerte-como por una camada surgida de la Academia Nacional de Bellas Artes en la que destaca Mario A. Canale, que sumo a la ensenanza del grabado en la academia la proctira junto al italiano Alfredo Bosco, estupendo agtafuertista, que publicaba la revista récnica El arte y la forogrofía en 1915. Canale fue el promotor de la Sociedad de Grabadores que editó, bajo su dirección, los tres nuimeros de la revista El Grobodo en 1916. con xilografias del mismo Canale, Hugo Garbarini, Antonio Sibellino, Nicolás Lamanna. Cayetano Donnis, Gregorio López Naguil y Raul Mazza. Los asuntos de El grabado son "populares": segadores, orquestas de tango, escenas de circo, etc, Esta revista tuwo amplia difusión en su momento por la actividad incansable de promociòn de Canale, y es dificil que Sabogal no la haya conocido, mas cuando en ella editaban sus iminas algunos compañeros de estudios.

24. Hulto an contra-nalos anteries en 1914, tamliẹn actwado por les artintas de-izquiendes.

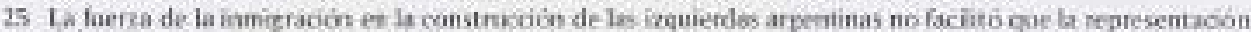

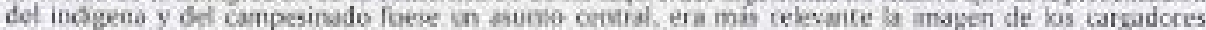
portuarios. Fovo cantio deste medisdos de los anos reinta con le discusión sobre an macionalidades opnmidas jaurque en la versión del comunismo kocil se equiparaba a las natwos con fos coiboos jution y. fundamentalimente, en los anses cincuensa cuando el campesaido fue considerado sujeta mevelucianano. 


\section{Segunda parte: el artista consagrado}

La inauguración, en las sslas de Amigos del Arte, de la Exposicion de pintara pernana; fost Sobrgal se postergo tua semana, hasta el 7 de septiembres a causa de la cantidad de obra a ser montadat 41 pinturas con motivos de Cuzcos, Lima, Htsancayo, Htanta y Puns, más 11 estampas de "estilo incaico" y 75 vilogratias subere Cuzco y Arequipa, que ocuparon las dos salas principales de la institución porteìa por 10 dias, tiempo habitual entonces de las muestras. El conjunto de grabados habia sido exhibido ances en Montevideo, con escasi tepercusion. Por el contrario la gran exposición en Buenos Áres consolidó la imagen de Sabogal como uno de los artistas centrales de la regíon para el público local y, a la vez. permitía confirmar la tesas de la posibilidad de la pintura de motivos americanos con len. guaje plastico moderno. It lugar elegido era ef apropiado. ya que desde la apertura de las nuevas salas en 1927 -más de trescientos metros cuadrados-Amigns del Arte, institucion privada fundada en 1924 por las damas de la alta burguesía portefia, se habia convertido en el espacio relevante de la cultura argentina donde se cruzaban el arte nuevo con el más tradiconal, el colect. cionismo privado con la difusión del arte internacional. los escritores y artistas de las. vanguardia formal rioplatense. con los artistas anarquistas $y$ comunstas que defendian el arte social. Fue el espacio hasta su cierre a principios de. los anos cuarenta-que combinaba el prestigio institucional con los negocios del mercado de arte, Exponer en Amigos del Arte era una excelente opción: no sólo los artistas pagaban una comisión sus. tantivamente menor por las veruas realizadas en el local snc que además sumaban ef mayor impacto de prensen por el prestigio "fitantcopico" de la institucion ${ }^{2}$.

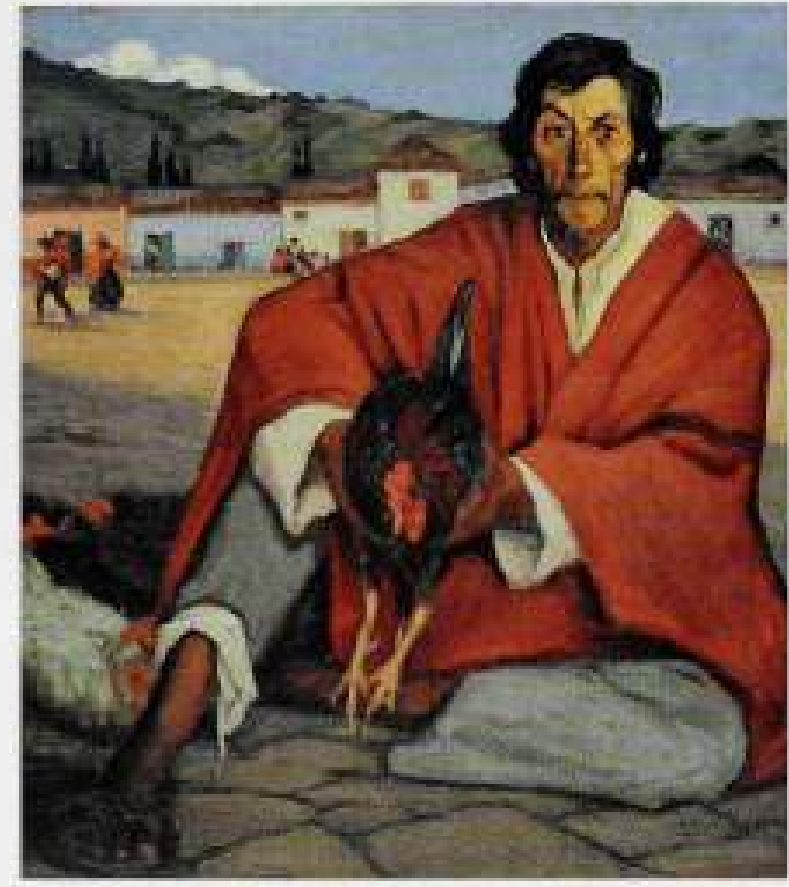

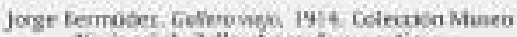
Naciens de Jellas Artes luerna Nino

Esta sociedad cultural habia demostrado siempre un fuerte interes por el arte americanop: La muestra de Sabogal se incocpora en esta linea constante, pero merece remarcarse la exposición, en el mismo año, de ochocientas piezas entre cerśmicas y textiles de "arqueologia perviana de Nazca y Trujillo" del Museo Frnográfico.

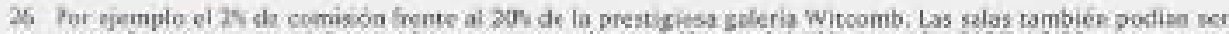

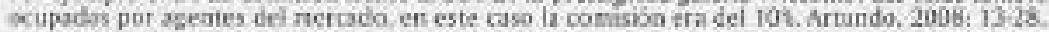

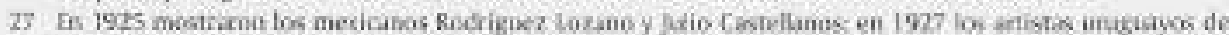

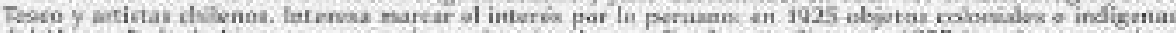

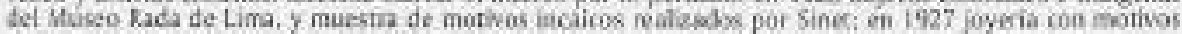
aneicanos de Saniago Cokzodine ibidem: 226227 


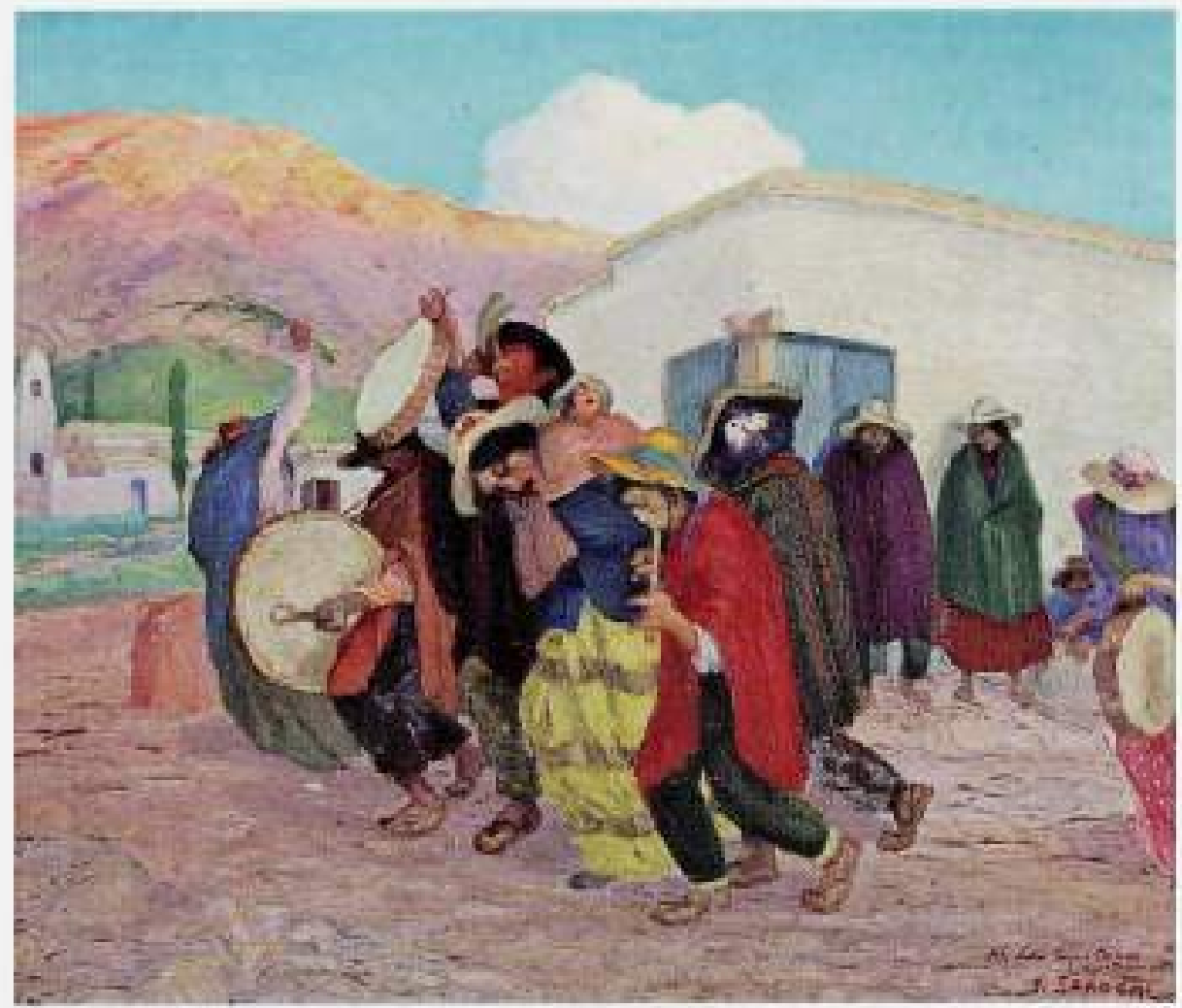

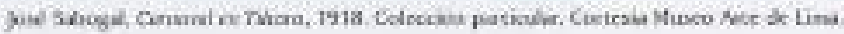

Sin cluda, 1928 fue uno de los inśs relevantes en la calidad y diversióad de las exposiciones en las salas de Florida 639. Entre ellas lat gran muestra de Pedro Figari, ocupando tres salas, que presentaba su singular lazo entre motivo tracticional y lenguaje plastico moderno; los frisos de Aifredo Gramajo Gutierrez, los pinturas de Alfredo Guido y los tejidos indigenas para el pabellón argentino de la Exposición lbero Americana de Sevilla. A la vez fue un ano clave para La difusión de los Artistas del Pueblo, que era ocro de los vinculos de Sabogal desde 1918, la extraordinaria muestra postuma de José Arato, la de Guillermo Facio Hebecquery las maquetas de teatro de Abraham Vigo. Estas menciones afirman que la muestri de Sabogal no actuaba extempoctaneamente en Buenos Nires. sino que permitia establecer filiaciones desde el propio calendario de Amigos de Arte. Dos muestas antológicas de gran importancia para la historia del arte argentinos se llevaron a cabo también en 1928, la primera de los artistas modemos conocidos como el Grupo de Paris (Huracio Bueler, Aquales Badi, Hector Basaldáa, Antono Beraí y Lino Enea Spilimbergo), y la segunda la de Cesareo Demaldo de Qui ros con su celebre serie de los gauchos entrerrianos que actuaron, desde entonces, conso definicion ideolsgica del arte nacional. Fsta ulrima antecedió a la de Sabogal con exite de pubibico notable. En las orras dos salas de Amigos del Arte, al mismo ticmpo que Sabogal, se montaron los paisajes estructuredos de fray Guillermo Butler-us dominico adicto a la pintura de palsaje, tanto de los rancones fraisceses como de los de los alrededores de sy convento 
en la sierras de Cóndoba-y los motivos gauchos del campo argentino del acuarelista Alberto Guiraldes".

La obra de Sabogal fue reproducida ampliamente en los diarios y revistas, en particular

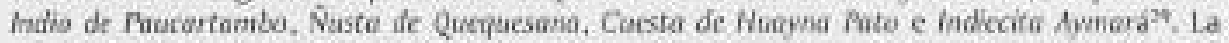
participación en el salón de los independientes de 1918 , et exito er Lima y la compra de su obra en México fue la información reiterada en diversos periódicos.

Sabogal era consciente del desaffo que planteaba seguir en la misma sala al exito de Quiros: "estaban tratados en intensa gama roja que necesariamente tenia que ser perjudicial para los expositores siguientes, $Y$ tal sucedió conmigo, al principio el publico encontná deswai. do mi cromatismo." La critica. sin embargo, comprendió otros aspectos de su obra. en particular la relación entre el paisaje y las figuras; si el primero seĥalaba "preocupaciones. del aire libre". las figuras eran "expresiones sintéticas cel carácter y el wolumen." "3i, arte "modemo, pero responsable" ta, ta obra de Sabogal furciono. entonces, como un punto medio: modernidad an radical, que abrevabo en la tradición aunque la sítesis-podia hacer perder la emación atribuible al indigena: el dolor, el sufrimiento, la quietud, etc. La estilización lo alejaba para la critica del impacto visual de pintores que spelaban a lo emocional. Una retorica más centrada en el ethos del discarso, que en lo patético de la forma, como. Quirós.

A regresar a Lima, Sabogal en una entrevista comenta que Victor Delhez había sido su prixcipal "prepagandisza". junto a las "atenciones" recibifas por Quirós" , Para Delhez. grabador belga y activo miembro de la Acción Católica, Sabogal era moderno en sus gzabados. la pintura todavia no habia logrado su forma*4.

Las vetitas fueron aceptables; entre ellas el Museo Nacional de Bellas Artes adguirió Cruz

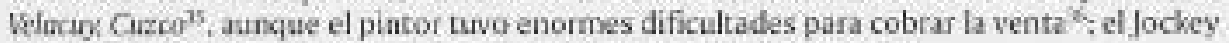
Club, el principal de la elice argentina, optó pot Cuesta de San Blias?.

Asi, Sabogal en 1928 fue cooptado por los defensores de la "tradiciōn", con su beneplacito al punto cue subraya que los artiscas argentimos siguen "la cordura tradicional" fusera del

28 A la inazouración de las tres exposiciunes de Amigos def Arte concurio el presidente Marzekr T de Avrat.

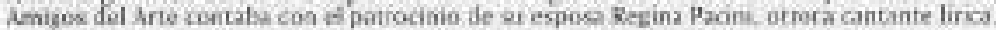

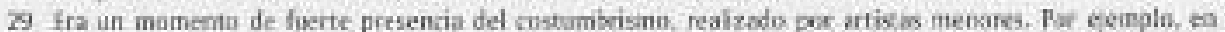

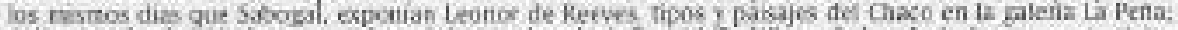

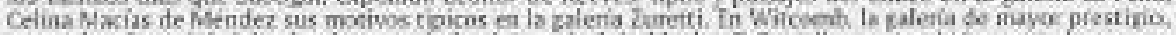

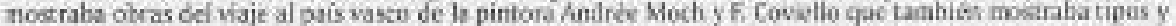

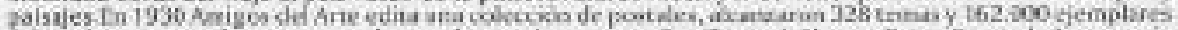

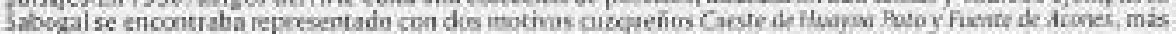

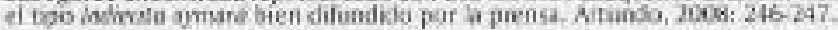

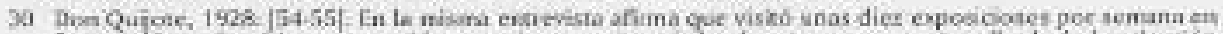

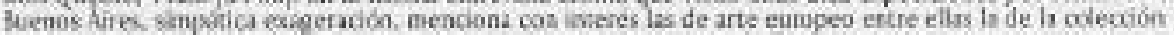
Gonvilez Garacon, que preservaba ef paraleto del arte invodervo cos el atricain

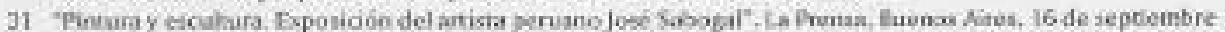
te $192 \mathrm{~K}$

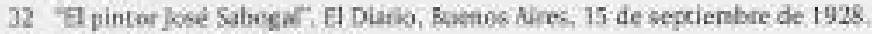

33 Don Qxace, isga: 5455 ).

34 Dethez, $1926,22$.

35 Gijlaf, 2010, if 237 .

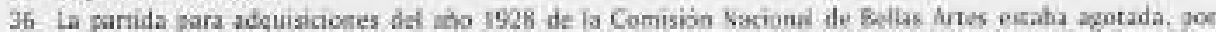

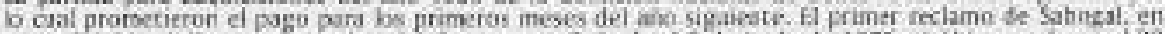
carta dotigita al diector Copertino det Campo esa fechada of 2 de junio de 1929, ex ultimn retamo el 23

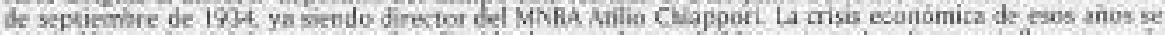

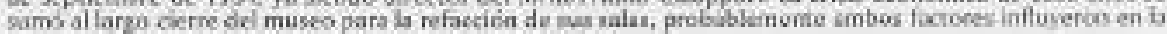
sorprenderve demura. Legajo de obra, MNBA. Aryenta

77. Perdica ea el incendia de ia sede el is de abri de 1953 . causodo por inarifestantes en roicrióa al atentadi antipsronista ocumde durinte un scto sindical ent la placa de Mayo 
impacto del futurismo, expresedo sobo por Emilio Pettoruti (Saboyal, sin embargo, conservaba un dibujo temptano de Pettorutil. Desde ya, los attistas nacionalistas podias llevarlo a sus filas, aunque su clara habia sido comentada por la prensa de los. más diversos signas. políticos". En tur contexte marcadio por los debates entre b "tradicion" y el "arte nuevo", La obra de Sabogal actua desde ocro discurso modemizador, el americanista. Demostraba la posibeidad de ana modernidad propia. Tal vez por eso el ataque furibundo y breve del arquitecto Alberto Prebisch desde la revista catolica Criterio, defensor del arte purn y la arquitectua mockema:

Si no peruana, como lo pretende el autor, esta pintura es, por lo menos, pésima. Supone asi la falaria de ciertas peregritas teorias americanistas en el arte. No es de la copia más o menos fiel de un motivo regional que ha de salir un are americano [.... el gran arte supera ef regionalismo anecóórico?

De este modo, la exposición de Sabogal en Buenos Aires, ya con su lenguaje artissico consolidado, fue un capitulo mis entre la disputa entre las vanguardias y la tradiciōn nacional. sin embargo un par de años después la politización creciente ante el avance del fascismo obligó, râpidamente, a poner otras discusiones e imágenes en escena ${ }^{20}$.

38. Vesse el listado de crikea en Majufy matiandes, 2013: 278-279.

39. Prebisw. 1926

40 Les grobadus de Sabegal tueson por la inquerdu cono acurtio en br exposidón de 1939 en el Tesers del

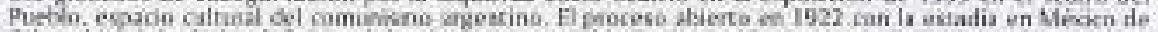

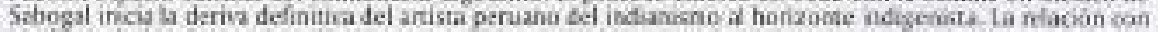

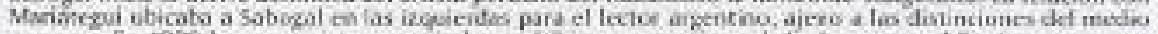
perusso. In 7959 los coenunstas neresirahon exhiber su potencia el la lucta contra ef fascismo, sumar a

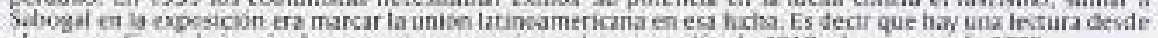
ef varteusodicno de inguierdas argentina anve eje es el conira-sabia de 3918 y la moestra de 1939. 


\section{BIBLOGRAFIA}

Amign. Roberto y Maria isahel Raldacarre

2006 Moescory discipulas. B arte argentina desdit of Antiwe Mariil A Canake Euepos Ares: Fundacion Eppigas.

\section{Amiso, Roberto}

2011 Crandes nuirtaras antentings $y$ Stimantrerixange de MNRA. Jose Saboyal. Evenos Aires: MN8A-Clatin.

\section{Artundo, Pacricia}

2005 Institución, arte y sociedad la Asociación Amizos del Arte. En Amigos def Ate 1924 1942. Buenes Aires MalbaFutadacioin Constastini.

Castillo Manuel hugel, Alfredo E. Latres y Jorge Sentibaitez (coords.).

2000 Mienaclon y fronteros. Mexico Norte Tivana: Eí Colegio de la Frontera Norte y Plaza y Valdés.

\section{Consejo Kacional de Educacioñ.}

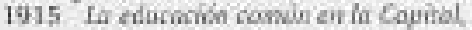
muwnsos y cerrichos nacianales Buenos Aires: Consejo Nacional de Educacion

\section{Delhez, Victor}

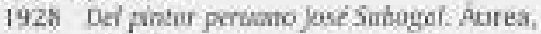
1527

\section{Don Quifoce [Solari, Carlos]} Socas de arte Fncrevista con Jesẻ Sabogar. Mhundial, Lima, año Vili, n, 439 [9 de noviembre de 1928) [34.55].

\section{Gálvez, Manuel}

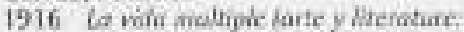
1910-1916). Buenos Arres: Sociedad cooperativa "Nosotros", 1916.

Higham, John

200211955 Strongers in the Land: Yatterns of Amsrimn Nativim, 1860-1925 Dustuen tiniwersity press

Folcon, jorge

1986 Jose Solorgal, Lima: Instituta Sabogal de Arte.

Ferras, Graciela Liliana

2007 Ricardo Rojass anmigración y bacioeses 4 Argentina del Centenario. Menowia E50otuded. (Pootifica Unversidad javeriana, Dogota) vol. 11, nuts, 22. wero - junco de 2007.

Kuoa Arce, Elizabeth, Rudrigo Guticires Viñuales, Ramùn Gutiórrex y Graciela Maria vintuales

20199 Cuzbo Buenux Airec Ruta de ba

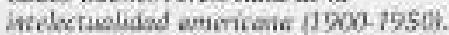
lima: Universidad San Martí de Porres.
Lauer. Mirlos

1998 Andes Wheguvarise. Disrursos del indigenismo 2. Cusco: CBC.

Mailuf. Nacalia

2010 lose Saboy, En Miseo Narimoin de Acties Artes. La caimción, Nmizon, Roberto idirec. I. 13: 237 Huenos Aires AAM NBh

Majhif. Natalis y Lris Eduandon Wuffarden 2012 Sobogal, tima: Museo de sarte de lima

\section{Muñuy, Migued}

Manuel Galvez, criticu de arte. Gaiana. Revista electrónica de Historia det Arte y Cultura Visual del Centro Argentino de liwestigadores de Arte (CA.MI. $\mathbf{N}^{\circ}$ ) Sinc 2012 , en lined desde ed 4 fallo 2012 hitpw whwcaiana org,ar

Malkarno, Martin

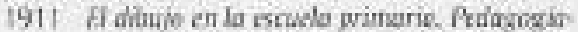
metodorogin. Buenos hires- Cabaur y Cla. fdicores.

\section{Peahos Marra}

1990 Notios en el solón. Artes plasticus e derkidad en fa primera mitad del sigio XX En Thas las pasos de in norsm. Sother Nacioncites de Deflas Artes (1911-1989). Penhos, Marta y Diana Wectsler ieds.l 111-152.41. Buenns Aires. CAIA Edxiones del ieguero.

\section{Bojas, Hicardo}

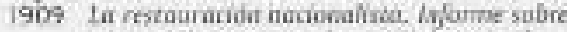
clucaciin. Butenos Aires Ministerio de justicial e Instrucción Pública.

\section{Prebisch, Alberis}

1928 Josè Sobrizal /Xmigos del Artel. Criferio, 20 -septertbre.

\section{Puggros, Adriana}

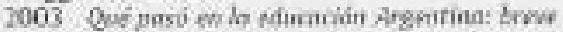
tichnria desde ie conquista hos'd ef prestrife. Buenos hines: Edicotia Galema.

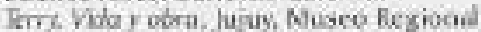
de Pintara "Jose A ferry". Secretana de Estado de Cultun. 1981

Torres Bohl, Jose

1489 Abuites sobre Jost sabogal; vida y abor. Lims: Hanco Central de Reservo del Pera

Wechoster, Diasa

1999 Salnoes y pondra clones. En Thes les pases de ia nomma. Sabites Nacianales de fellos Artes fl912-7989 Pethos, Narta y Disia Wuchsfer (eds.): $41-80$. Buenos. Aires, CAla-Edicioges del likuero.

Wiesse Maria

1957 fosé Sobogol, ef artisro y el hamber, tima: Compania de impresiones y publicidad. 